Proceodings of the 1992 IEEE

Intemational Conforence on Robotics and Autanation

Nice, France - May 1992

\title{
AUTONOMOUS PLANETARY ROVER (V.A.P.) : ON-BOARD PERCEPTION SYSTEM CONCEPT AND STEREOVISION BY CORRELATION APPROACH
}

\author{
L.Boissier, B.Hotz ${ }^{*}$, C.Proy - Centre National d'Etudes Spatiales (C.N.E.S.) \\ 18, Avenue E.Belin 31055 Toulouse Cedex - France \\ O.Faugeras, P.Fua - I.N.R.I.A./Sophia-Antipolis. \\ 2004, Route des Lucioles, B.P. 109 - 06561 Valbonne Cedex - France
}

\begin{abstract}
This paper presents a little part of the work currently developed at CEA, CNRS, INRIA, ONERA and CNES in the framework of the Autonomous Planetary Rover program (V.A.P.). In the first part of the paper, a system concept approach for the VAP application is quickly presented. The second part is a special focus on the V.A.P. Perception System where we discuss and show some of the results obtained with the stereovision by correlation algorithm on outdoor simulated Mars terrain scenes.
\end{abstract}

\section{Introduction}

The French Space Agency (CNES) defined and launched in 1989 the "Autonomous Planetary Rover" program (V.A.P.) [1]. The main research theme concerns a Mars rover but other themes, including possible missions to the Moon, are considered for future developments. In 1990 and 1991, CNES and a consortium of the main french robotics research laboratories involved in terrestrial mobile robotics (CEA, CNRS, INRIA and ONERA formed the RISP** group) have carried out R\&D studies identified as critical due to the dramatic originality of Autonomous Planetary Rovers. Of paramount importance to the planetary exploration missions is the trade-off which must be made between the robot's decisional and operational autonomy, and the on-board resource requirements (processors, implementation, energy, etc.). A second key element closely linked to the preceding one concerns the distribution of the sensory and decisional "intelligence" functions among the various physical systems connected via channels of communication with limited bandwidth, time delays, etc.

Acknowledgements. This work has been supported by the CNES and INRIA within the framework of the V.A.P. program of the French Space Agency (CNES). * Currently researcher at INRIASophia-Antipolis. ** R.I.S.P.: Intervention Robots for Planetary Exploration
The overall program covers all the topics necessary for the design of an intervention robot such as a Mars rover. So, within the framework of Mars exploration, due to the significant time delay transmission and to terrain characteristics, the robotic challenge is to provide the highest degree of autonomy to the planetary rover [2].

In this paper, we try first to give a quick overview of a typical mission and of the associated system concept. In a second part, we describe the last improvements and associated tests results of a stereovision algorithm based on correlation technique.

\section{System Concept for a Mission on Mars}

The overall mission system consists of 3 segments: the Earth segment, the Mars Rover segment and the Mars orbiting segment. This last segment is a spacecraft dedicated to scientific experiments, which provides stereo imaging of the area covered by the rover.

The main design problems of a Mars rover can be best understood in terms of typical missions. Once safely on Mars, the rover is expected to be operational for about one year and to carry on 8 hours per day activities (figure 1). Although missions will be slightly different every day, there will likely include the following 3 parts: (i) worksite experiments, such as sample collection or measurements, (ii) moving to the next site with autonomous navigation, involving natural landmark recognition, inertial localization, environment modelling for path planning or obstacle avoidance, real-time on-board decision-making to change, if necessary, from an initial route to an other one..., (iii) worksite modelling and data transmission to Earth to prepare a mission plan for the next day.

Each day therefore begins with the downlosding of a task-level teleprogram from Earth. Figure 1 attempts to give an idea of a typical mission execution with a coarse grid representation of the rover total mission area and a finer grid representation of the initial local environment such as previously perceived by the robot.

The rover area stereo images from the orbiting segment are processed on Earth to build a Digital Elevation Map (DEM). 
This map and the rover area images are then computed to provide the rover with a bitmap and symbolic feature based map including landmark models. This map is focused over the network of routes that have been computed on Earth. This set of routes is established so that the rover can reach the goal-site from its initial location, taking into account criteria such as travel path length, vehicle safety (roll and pitch thresholds). The map and associated informations are finally downloaded to the rover during the tasklevel teleprogramming phase.

The general functional organization of V.A.P. is composed of two distinct systems: the Operator Station and the onboard Robot Control System [3]. The Operator Station provides the necessary functions to allow a human operator to build an executable mission (i.e. a mission that can be interpreted by the RCS) and to supervise its execution. The process of building an executable mission is decomposed into two phases which correspond to two different levels of abstractions and to different planning techniques: mission planning, and task-level teleprogramming. Then the executable mission, i.e. the task-level program, the bitmap and symbolic terrain feature based map, a set of possible routes, is sent to the Robot Control System where interpretation and execution of each elementary task is completely on-board controlled without any direct interaction with the operator. This provides the rover with operating autonomy at task level [4].

Therefore the environment perception and modelling is one of the main domain to study and improve.

\section{V.A.P. Perception System}

The perception system achieves different functions according to the place where the processes are done, i.e on-Earth or on-boand.

In this paper, we will focus on the on-board perception system but more details on the on-Earth processes are given in [5] and [6].

\subsection{On-Board Perception System}

The on-board perception system accomplishes the following functions:

- internal and external vehicle safety including obstacles

avoidance and hazardous areas detection,

- localization estimation based on landmarks recognition and horizon profile matching,

. vision feedback control of the rover manipulator for sample collecting,

.3D scene modelling based on elaboration of a local DEM and then extraction and labelling of the most important features of the terrain,

- world modelling based on fusion of scenes, along panoramic displacement of the perception device, and along robot motion.
The V.A.P. on-board perception device is composed of two or three $C C D$ cameras and a laser device for range measurements. This device should be either a laser range finder mounted on a scanning platform - minimal version - or a space qualified laser camera. The processing of their measurements gives depth information by means of stereovision techniques or direct depth measurements. In addition, to increase its local field of view, the perception device uses pan and tilt facilities. Studies are under progress to analyze the technical feasibility of a "perception mast" and its contribution to upgrade the on-board environment perception system. The redundancy function attached to the rover manipulator which is equipped with a CCD camera is also under analysis. To cope with the safety vehicle requirements and to build the internal robot state, the perception system needs some dedicated devices to measure roll, pitch, yaw variations as well as propulsion strain due to terrain resistance, etc.

Current V.A.P. research developments on stereovision techniques focus on trinocular stereovision by contour matching and correlation techniques. An overview of these techniques is available in [7]. In the following sections, we present developments of a stereovision algorithm suited to unstructured environment and textured surfaces, based on a correlation technique and we present the last technical improvements and associated results obtained on image rectification, estimation of correlation accuracy; tests are made using outdoor simulated Martian obstacles.

\subsection{Trinocular Rectification}

Given several images and a point in one of them, the corresponding points in the other images are bound to lie on epipolar lines. To optimize stereovision by correlation, first a rectification process is needed to obtain parallel epipolar lines. For three cameras, this consists in reprojecting the images to a plane that is parallel to the one passing by the three optical centers (figure 2).

An important constraint is to minimize image distortions, to make sure that the correlation algorithm will run correctly. We implemented the rectification method presented in [8] that simplifies the laying of epipolar lines (fig. 3) so that: - a point from image 1 find its corresponding points on the same line in image 2 and on the same column in image 3 ; - a point on column $U$ in image 2 corresponds to a point on line $V$ in image 3 with $U=V$.

The reference vectors $U$ and $V$ in the rectified plane verifying these conditions are shown in figure 4 . When we straighten the images, important and quite different distortions are obtained, probably due to the relation between image 2 and 3 because V $3=\mathrm{U} 2$.

Suppressing this relation enables to take the same reference vectors $\mathrm{U}$ and $\mathrm{V}$ for the three rectified images (figure 5). The mathematical proof is detailed in [9]. These vectors are 
respectively collinear to $\mathrm{C1C} 2$ and $\mathrm{C1C} 3$, which form an angle near 90 degrees in our case, so that the distortions of the rectified images will be similar and minimized (figure 6). It is true that this modification prevents from correlating images 2 and 3 together easily, but up to now, the tests were limited to correlation between images 1 and 2 with horizontal epipolar lines and between images 1 and 3 with vertical epipolar lines and this approach seems to be good enough .

Finally, the rectification matrix is obtained by giving the image coordinates of a point in space in two steps: first of all by computing the path from a $3 \mathrm{D}$ point to the plane containing the three rectified images, and secondly by transforming the obtained coordinates into the image coordinates according to the choice of the reference vectors for these images. More details are available in [9].

\subsection{Stereovision by Correlation}

The correlation algorithm developed for the Autonomous Planetary Rover (V.A.P.) is presented in [10]. It compares for each point of image 1 the grey levels on square windows along its corresponding epipolar line in image 2; the most similar window according to a given criterium is kept, its center designates the corresponding point in image 2 , and the difference of range between the two matched points is stored as the disparity value. To check the validity of the correlation, the same process is applied on the other way from image 2 to image 1 and the result is validated only if the two disparity values are similar; otherwise no correlation has been found for this point and the result appears in black. For each point of image 1 disparity value is associated, which, thanks to the previous calibration, can be transformed in a depth value or in a 3D point. This stereovision by correlation method has been tested on an outdoor simulated Mars terrain to evaluate its precision and its robustness, and to choose the better parameters of the correlation algorithm.

\section{Description of test-bed}

The outdoor simulation of Mars terrain covers 16 square meters, with rocks from 0.4 to 1.2 meters high and with some sand material (figure 6). A mast with three CCD cameras (12.5 $\mathrm{mm}$ focal length) in L-configuration and distant of $40 \mathrm{~cm}$ from each other is mounted on the mobile robot HILARE 1.5; the system is calibrated using a plane sighting marks in two parallel positions. Several views of the scene with various solar light conditions and from various ranges were acquired.

To evaluate the correlation accuracy, we need to be able to compare disparities obtained by the correlation algorithm to "actual" disparities and this for a high number of points. To do so, two hundred marks were fixed at random on the outdoor simulated Mars terrain. Images of the scene with marks enable human visual matching of marks on the three images; the disparities obtained like this are considered as a reference and compared to the results of the correlation method on the images without marks.

\section{Choice of parameters for correlation}

Two parameters must be chosen carefully to obtain good results: the correlation criterium and the size of the square window. For the tests, the square windows range from $3 * 3$ to $19 * 19$ pixels and the following correlation criteria are used:

$I_{1}$ and $I_{2}$ are the intensities of images 1 and $2, I_{1}$ and $I_{2}$ are the mean of grey levels on the window, $d$ is the tested disparity, $x$ and $y$ are the coordinates of the point in image 1 .

$C_{1}(x, y, d)=\frac{\sum_{i} \sum_{j}\left[I_{1}(x+i, y+j)-I_{2}(x+d+i, y+j)\right]^{2}}{\sqrt{\sum_{i} \sum_{i} I_{1}^{2}(x+i, y+j)} \sqrt{\sum_{i} \sum_{1} I_{2}^{2}(x+d+i, y+j)}}$

$$
C_{2}(x, y, d)=\frac{\sum_{i} \sum_{j}\left(I_{1}(x+i, y+j) \cdot l_{2}(x+d+i, y+j)\right)}{\sqrt{\sum_{i} \sum_{j} l_{1}^{2}(x+i, y+j)} \sqrt{\sum_{i} \sum_{i} l_{2}^{2}(x+d+i, y+j)}}
$$

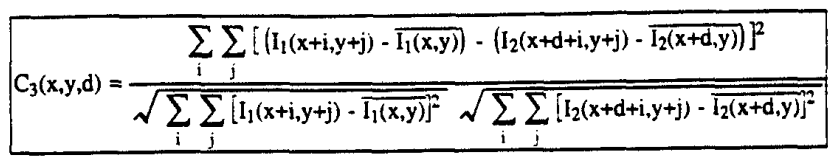

$C_{4}(x, y, d)=\frac{\sum_{i} \sum_{j}\left(I_{1}(x+i, y+j)-\overline{I_{1}(x, y)}\right) \cdot\left(I_{2}(x+d+i, y+j)-\overline{I_{2}(x+d, y)}\right)}{\sqrt{\sum_{i} \sum_{j}\left[I_{1}(x+i, y+j)-\overline{\left.I_{1}(x, y)\right]^{2}}\right.}} \sqrt{\sum_{i} \sum_{j}\left[I_{2}(x+d+i, y+j)-\overline{\left.\bar{I}_{2}(x+d, y)\right]^{2}}\right.}$

To find out the better correlation criterium and window size, tree quantitative values are used: the percentage of correlated points on the overall image and for several window sizes, the same percentage but limited to the 200 marked points and, the disparity error which is the mean error between the human visual matching disparity and the computed disparity on the 200 reference points.

Figure 7 shows the results of tests and leads to conclude that on one hand, the best window sizes are $5 * 5$ and $7 * 7$ because they form a good compromise between precision and density of correlation and that on the other hand, the correlation criteria number 2,3 and 4 give good and rather similar results.

\section{Precision and robustness of correlation}

The percentage of correlated points on the overall image is about 80 $\%$ for the 7*7 windows. If we look now the percentage of correlated points on the 200 reference points, the wall in the background of the scene which is a smooth surface not representative of the Mars terrain is suppressed and the proportion of correlated points reaches 
$95 \%$, which is a very high score (figure 7a). The density of the disparity map can be checked on figure 8 , which is rather smooth, and where almost all the rocky area has been correlated without obvious errors.

Therefore, the disparity error is low (figure $7 \mathrm{~b}$ ) : the best precision is obtained for $5 * 5$ windows (an error value under the pixel is founded for $90 \%$ of the 200 marked points) and the mean error is still under the pixel for $7 * 7$ windows. We must precise that a disparity of one pixel corresponds roughly to 10 centimeters in depth in this experiment, and that this type of scene is about 6 meters distant from the mobile robot.

During the tests, the robustness of the correlation method was checked from other points of views and under various solar light conditions. In some cases, a few problems occurre on sand, on shadowed areas, and on occlusions, but the method still stands to be reliable because it does not give errors: these types of areas are declared as not correlated and correspond to black areas in the disparity map.

\section{Implementation}

The computing time of the algorithm has been highly optimized: it takes 48 s for a $256 \times 256$ grid, and a disparity search interval of 20 pixels on a Sun 4 with a Sparc processor at $40 \mathrm{Mhz}$ and $30 \mathrm{Mo}$ of memory. We also tried an architecture based on four DSP processors and we obtained about $6 \mathrm{~s}$ under the same conditions.

\section{Conclusions}

We have briefly presented the Autonomous Planetary Rover (V.A.P.) system concept which stresses two essential aspects for a machine to qualify for a mission such as Mars exploration and scientific experiments:

- the capacity to have different tasks to be assigned by an Earth based operator, and

- the necessity for the rover to exhibit operational and decisional autonomy.

The on-board perception system functionalities and components which correspond to this concept have been defined, as well as the tests results of the stereovision by correlation algorithm have been presented and discussed. The Autonomous Planetary Rover has now a processing sequence for on-board stereovision by correlation that seems to be well adapted to its environment. This outdoor simulated Mars terrain has been used to acquire a lot of stereovision images but depth data were measured with a 3D laser camera, too. Additional experiments must be done: contribution of the third camera (particularly to solve occlusion problems), interest of using higher points of views, etc...In addition, the next studies will focus on cooperation between stereovision and laser (sensor's configuration, cooperative strategy, fusion data process...), and on implementation of the correlation process on other specialized parallel image processing architectures, too.

The V.A.P. program is now entering a new phase which comprises: a) further developments on some of the sub-systems concepts, b) hardware and software developments of the sub-systems that constitute the rover backbone and for this phase, CNES envisions two important program extensions: the participation of national industry for point (b), and reinforced international collaboration.

\section{References}

[1] D.JP.Moura,F.Rocard,S.Coutin-Faye,L.Boissier, G.Giralt. The French preparatory program for future Mars rover mission. 41st congress of the Intemational Astronautical Federation, Dresden (Germany), October 90.

[2] L.Boissier,G.Giralt. Autonomous Planetary Rover: The Robotic Concepts. International Advanced Robotics Programme (LARP) Workshop on Robotics In Space, Pisa (Italy), June 91.

[3] R.Chatila, R.Alami and Al, Integrated Planing and Execution Control of Autonomous Robot Actions. IEEE 92 International Conference on Robotics and Autiomation, Nice (France), May 92.

[4] G.Giralt,R.Alami,R.Chatila,P.Freedman. Remote operated Autonomous robots. Intemational Symposium on Intelligent Robotics, pp 416, Bangalore (India), January 91.

[5] P.Julien,B.Rouge. Martian digital elevation (3D) modeling. ESTEC Workshop on Computer Vision and image processing for spaceborne applications, Noordwijk (Netherlands), June 91.

[6] P.Garnesson,M.Berthod. Planetary vehicle location. ESTEC Workshop on Computer Vision and image processing for spacebome applications, Noordwijk (Netherlands), June 91.

[7] C.Proy,L.Boissier,P.Fua,O.Faugeras. On-board perception for planetary rover. ESTEC Workshop on Computer Vision and image processing for spaceborme applications, Noordwijk (Netherlands), June 91 .

[8] N.Ayache,C.Hansen. Rectification of images for binocular and trinocular stereovision. Ninth Intemational Conference on Pattern Recognition, pp 11-16, Roma (Italy), November 88.

[9] B.Hotz. Etude de techniques de stéréovision par corrélation application au programme VAP. CNES technical document te/ae/se/sr 91/242, August 91.

[10] P.Fua. A parallel stereo algorithm that produces dense depth maps and preserves images features. Research report $n^{\circ} 1369$ INRIA-Sophia-Antipolis (France), January 91. 


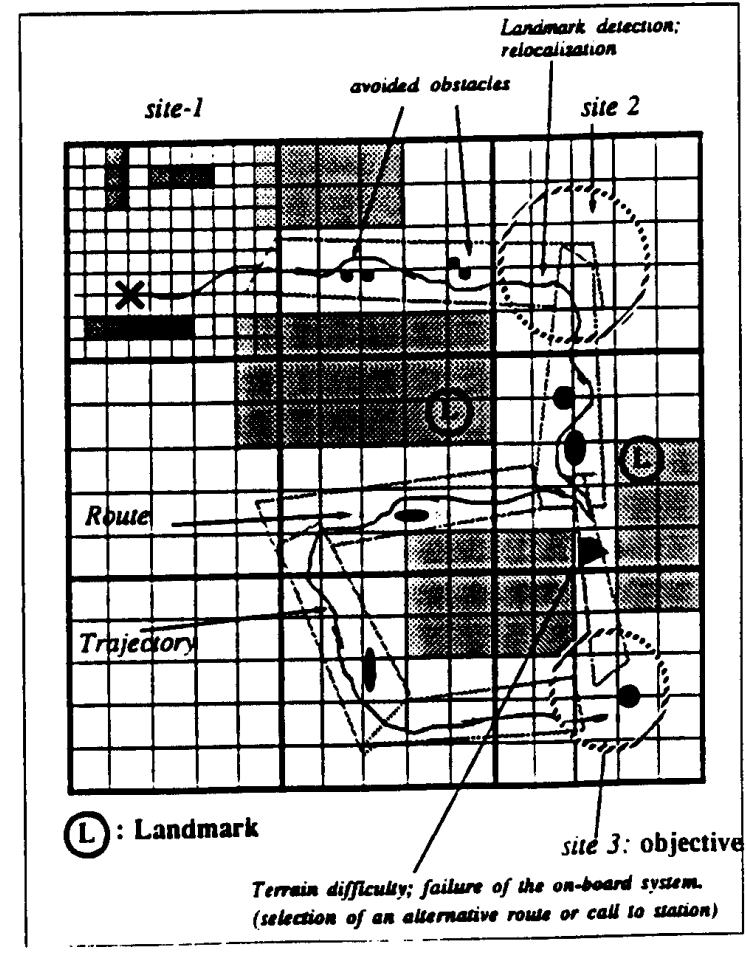

Figure 1: Mission execution

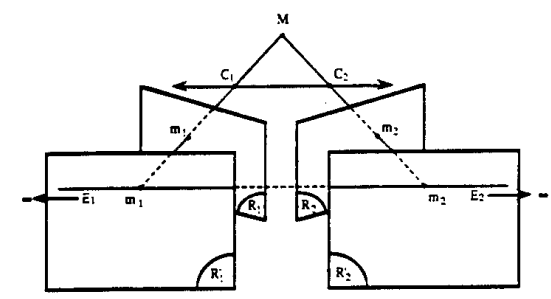

Figure 2: binocular rectification: projected images

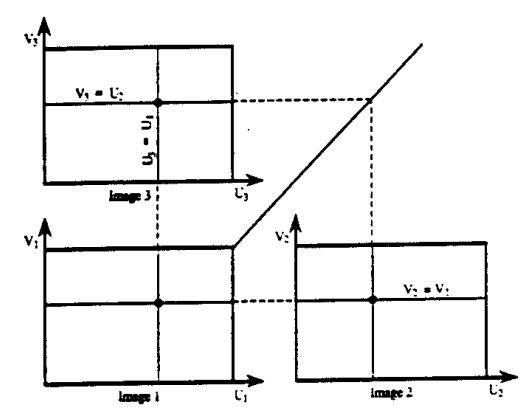

Figure 3: relations between points for trinocular rectification.

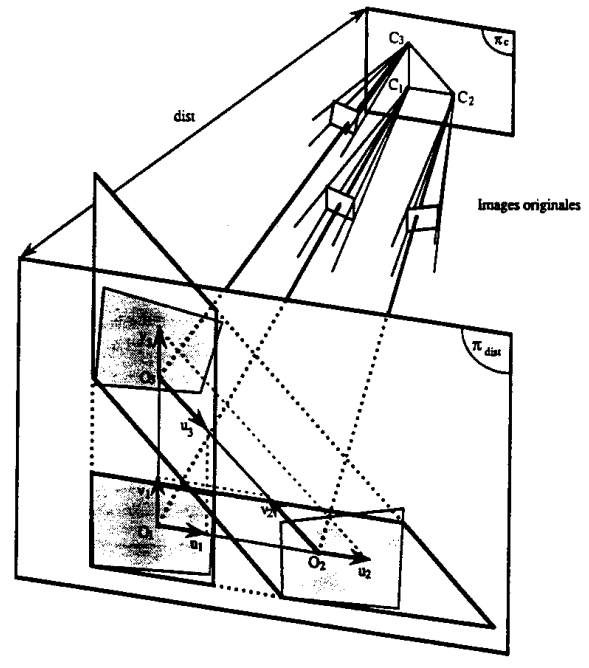

Figure 4: distortion due to the Ayache and Hansen method.

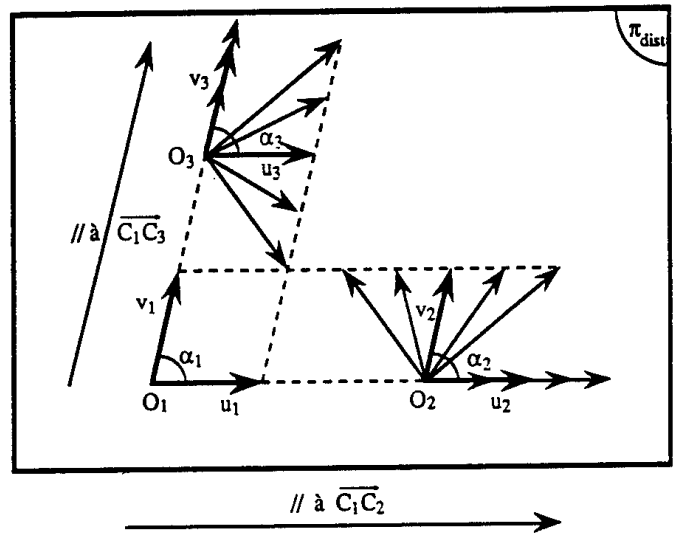

Figure 5: choice of reference vectors for the three rectified images. 

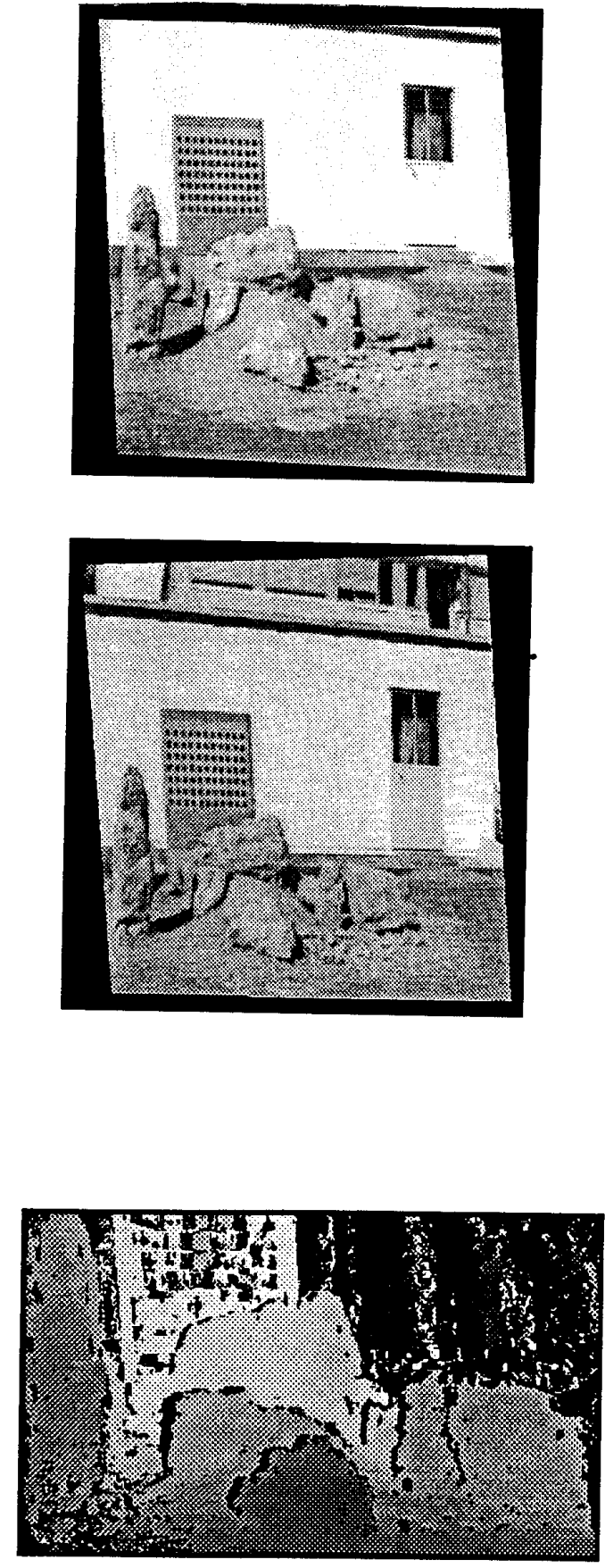

Figure 8: Example of disparity map obtained for $7 * 7$ windows and the second criterium
Figure 6: example of trinocular rectification applied on three CCD views of our simulated terrain.

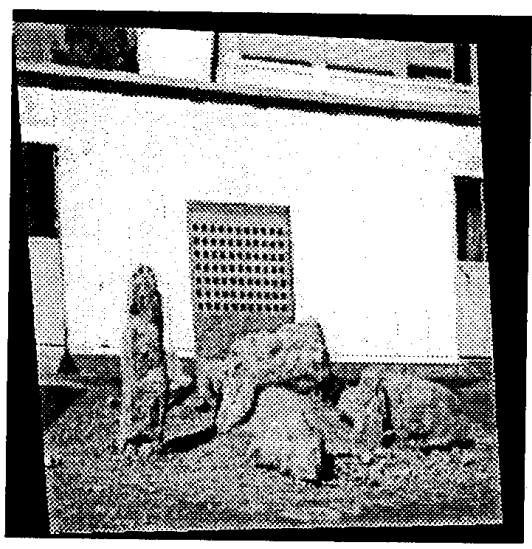

(b)

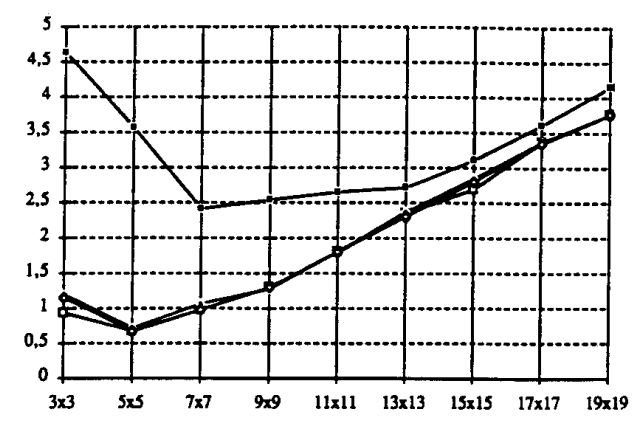

a. Crimen 2

- Criven

(a)

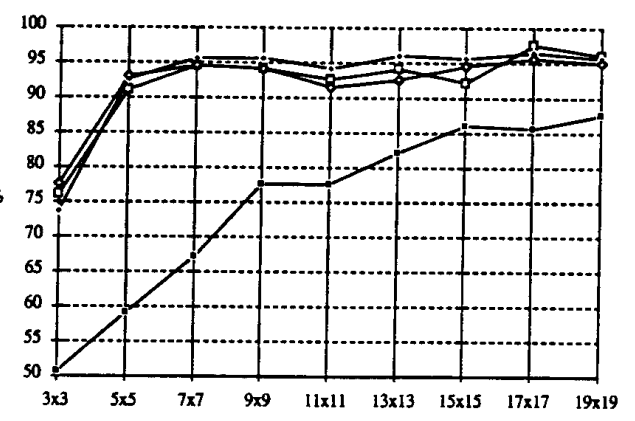

Figure 7: Curve of the percentage of correlated points (a) and of disparity errors in pixels (b) on the 200 reference points as a function of window size and for the four criteria. 